\title{
Concurrent hereditary haemochromatosis and idiopathic Parkinson's disease: a case report series
}

\author{
D J Costello, S L Walsh, H J Harrington, C H Walsh
}

J Neurol Neurosurg Psychiatry 2004;75:631-633. doi: 10.1136/jnnp.2003.027441

Hereditary haemochromatosis $(\mathrm{HH})$ is a genetic disorder in which abnormal iron handling leads to excessive iron accumulation in systemic tissues. Magnetic resonance imaging studies suggest excess iron deposition in the basal ganglia of patients with $\mathrm{HH}$. The symptoms of neurological complications of $\mathrm{HH}$ include cognitive decline, gait difficulties, cerebellar ataxia, and extrapyramidal dysfunction, but idiopathic Parkinson's disease, in which brain iron deposition is normal, has not been reported. We describe four patients with concurrent $\mathrm{HH}$ and IPD. Although three of the cases had risk factors for cerebrovascular and cardiovascular disease, computed tomography did not show ischaemic changes in the basal ganglia. We speculate that in these cases, abnormal deposition of iron in the basal ganglia induced the symptoms of IPD.

$\mathrm{H}$ ereditary haemochromatosis $(\mathrm{HH})$ is an autosomal recessive disorder characterised by abnormal iron handling, leading to excessive iron accumulation in systemic tissues. Although neuropathological evidence of brain iron accumulation is lacking, recent magnetic resonance imaging (MRI) findings suggest excess iron deposition in the basal ganglia of patients with $\mathrm{HH}{ }^{1}{ }^{1}$ Reported neurological complications of $\mathrm{HH}$ are clinically diverse..$^{2-9}$ These include cognitive decline, gait difficulties, cerebellar ataxia, and extrapyramidal dysfunction. Idiopathic Parkinson's disease (IPD), in which brain iron deposition is abnormal, has not been reported in patients with confirmed HH.

We report four patients with concurrent HH and IPD. We speculate that $\mathrm{HH}$ might result in excess brain iron deposition, which may contribute to the development of IPD.

\section{CASES}

Case 1

A 55 year old male with a past history of hypertension and Type II diabetes mellitus (DM) was found to have elevated serum ferritin on routine testing. HH was diagnosed based on the presence of excessive iron stores without cirrhosis on liver biopsy and homozygosity for the C282Y mutation. Serum caeruloplasmin and copper studies and computed tomography (CT) brain were normal. Within months of the diagnosis of $\mathrm{HH}$, he developed right upper limb resting tremor followed by rigidity, bradykinesia and hypophonia. The parkinsonism became bilateral within a number of years. A diagnosis of IPD was made based on the characteristic resting tremor, bradykinesia, rigidity and asymmetric onset. He responded well to L-dopa. Serial venesection did not improve his parkinsonism. Over the following 12 years his condition has progressed steadily but remains typical of IPD.

\section{Case 2}

A male patient with well controlled hypertension treated with metolazone and $\alpha$-methyldopa developed Type II DM at
49 years of age. His father died from hepatocellular carcinoma and a brother had type II DM. In 1983, haemochromatosis was suspected because of abnormal skin pigmentation at the age of 60 years and confirmed by an elevated serum ferritin, increased transferrin saturation, and increased iron stores with micronodular cirrhosis on liver biopsy. DNA analysis of the HFE gene was unavailable but HLA typing revealed an A2A3B7B12 haplotype, consistent with $\mathrm{HH}$. Serial venesections resulted in correction of the iron overload. When first seen by us at the age of 61 years, signs of IPD were established, with bradykinesia, bilateral upper limb rest tremor, and limb rigidity. $\alpha$-Methyldopa was discontinued and L-dopa subsequently begun, with a good response. There were no atypical clinical features at initial assessment or in the subsequent follow up years. He developed lower limb dyskinesias after long term L-dopa use. A brain CT scan revealed atrophic changes, without evidence of vascular disease. He died aged 66 years. An autopsy was not carried out and thus the neuropathological features of IPD were not confirmed.

\section{Case 3}

A 63 year old male presented with a 6 month history of lower limb and body tremor, hypophonia, and subjective gait instability. A deceased uncle had parkinsonism of unknown aetiology and a twin brother with HH was neurologically normal. Physical signs included bradykinesia, flexed posture, hypomimia, lower limb and truncal rest tremor, and limb rigidity. A diagnosis of IPD was made. There was a good response to L-dopa. A brain MRI was normal. Seven years after the diagnosis of IPD, investigation of abnormal liver function tests revealed an elevated serum ferritin, and subsequent genetic testing revealed homozygosity for the $\mathrm{C} 282 \mathrm{Y}$ mutation. A liver biopsy showed advanced siderosis without cirrhosis. Serum caeruloplasmin and copper studies were normal. His parkinsonism has progressed slowly without atypical features and a good response to L-dopa has been maintained.

\section{Case 4}

A 49 year old female was found to have Type II DM and hypertension in 1988. A brother had HH. Screening investigations confirmed systemic iron overload with elevated serum ferritin, and a liver biopsy showed heavy deposits of stainable iron without evidence of cirrhosis. Genetic testing revealed her to be homozygous for the C282Y mutation. Serum caeruloplasmin and copper studies were normal. The iron overload was corrected by venesection. Between 1994 and 2000, she presented with intermittent claudication, unstable angina, and three episodes of cerebral ischaemia.

\footnotetext{
Abbreviations: $\mathrm{CT}$, computed tomography; $\mathrm{HH}$, hereditary haemochromatosis, IPD, idiopathic Parkinson's disease; MRI, magnetic resonance imaging
} 
Sequential CT scans revealed evidence of a right middle cerebral and right posterior cerebral artery infarcts. In August 2001, she developed a "pill-rolling" right upper limb tremor. Examination revealed a right upper limb rest tremor, asymmetrical upper limb rigidity, hypomimia, bradykinesia, and flexed posture. A further brain CT, particularly of the basal ganglia, revealed no new infarct. Despite the cerebrovascular disease evident on imaging, she does not have the characteristic features of "vascular" parkinsonism, namely "lower body" parkinsonism, prominent or early gait disturbance, lack of tremor, lack of response to L-dopa, advanced age at onset, prominent early postural disturbance, dementia, corticospinal tract signs, pseudobulbar palsy, or any other clinical correlates of "vascular" parkinsonism. There was a good response to L-dopa. A diagnosis of IPD was made based on asymmetric onset, rest tremor, bradykinesia, and rigidity without development of atypical features over time.

\section{DISCUSSION}

To our knowledge, this is the first report of concurrent $\mathrm{HH}$ and IPD.

These cases were recruited from a population of approximately $180 \mathrm{HH}$ patients attending an endocrine clinic. We found a greater than expected number of IPD patients in this $\mathrm{HH}$ population. As the prevalence of IPD in the general population is approximately 1:1000 under the age of 65 years, ${ }^{10}$ and the majority of our $\mathrm{HH}$ patients $(68 \%)$ were under this age, we would expect no more than one case of IPD in this population of $\mathrm{HH}$ patients. Despite this, these cases may represent the chance occurrence of two common diseases.

In our cases, while there was no fixed chronological relationship between the diagnoses, it seems likely that the diagnosis of HH lay undetected for several years. Cases 2 and 4 developed IPD 12 and 13 years respectively after diagnosis of $\mathrm{HH}$. In cases 1 and 3, it is likely that systemic iron overload was present for some years before either diagnosis, given the hepatic siderosis confirmed by biopsy. Both diseases have a considerable incubation time during which pathological progression is clinically silent. The average age at diagnosis of $\mathrm{HH}$ was 58.5 years (range 49-70), while the average age at diagnosis of IPD was 60.25 years (range 55-63). Only in case 3 did the neurological manifestations precede the diagnosis of $\mathrm{HH}$. Detection of $\mathrm{HH}$ in these cases was through family screening and serendipity. In the general HH population the average time from symptom onset to diagnosis of $\mathrm{HH}$ is 10 years. ${ }^{11}$ Therefore, development of $\mathrm{HH}$ probably preceded development of neurological symptoms in these four cases, including case 3. Of importance was the presence of systemic iron overload in all.

There have been three previous reports of concurrent parkinsonism and $\mathrm{HH}^{2-4}$ These cases exhibited diverse clinical signs such as cognitive decline, fluctuating level of consciousness, ataxia, pyramidal deficits, and parkinsonism, therefore clearly not representing IPD. In contrast, our cases are characterised by typical age of onset, typical symptoms and signs, L-dopa responsiveness, gradual progression and no atypical features such as early postural instability, early freezing phenomena, hallucinations unrelated to medications in first 3 years, early dementia preceding motor symptoms, supranuclear gaze palsy, slowing of vertical saccades, or severe symptomatic dysautonomia unrelated to medications.

We acknowledge the presence of possible confounding factors in some of the cases. Cases 1 and 2 had risk factors for cerebrovascular disease (hypertension and Type II DM) but brain $\mathrm{CT}$ in both showed no evidence of cerebral ischaemia. Case 4 similarly had risk factors for atherosclerosis but brain CT did not show evidence of ischaemic changes within the left basal ganglia. In case 2 , following $\alpha$-methyldopa withdrawal, the clinical signs persisted and progressed in a fashion typical of IPD rather than drug induced parkinsonism. None of the cases was taking psychotropic medications nor had neurological signs of liver disease.

Pathological accumulation of iron in systemic tissues results in organ damage. Physiological storage of iron within the body is maximal in the liver and brain. Iron deposition in the brain is highly regionalised, ${ }^{12}$ mainly in organic forms such as ferritin, ${ }^{13}{ }^{14}$ with little in free, reactive forms. Excessive accumulation of iron in a free state or release of physiological cellular stores of iron can lead to oxidative stress, ultimately leading to cell death. ${ }^{15}{ }^{16}$ There are three well defined neurological disorders in which brain iron overload is the immediate cause of neuronal damage. In pantothenate kinase associated neurodegeneration, an autosomal recessive neurodegenerative disorder, ${ }^{17}$ pathological iron accumulation occurs in the basal ganglia, resulting in extrapyramidal signs, dementia, and premature death. Acaeruloplasminaemia is an autosomal recessive condition characterised by complete caeruloplasmin deficiency, pathological brain iron accumulation, extrapyramidal signs, cerebellar ataxia, retinal degeneration, and progressive dementia. Caeruloplasmin is probably involved in cellular iron turnover and, when absent, pathological intracellular iron accumulation occurs. ${ }^{18-20}$ Neuroferritinopathy, a recently described autosomal dominant disorder, is characterised by late onset extrapyramidal disease, ${ }^{21}$ choreoathetoid movements, and parkinsonism. Brain histochemistry reveals pathological deposits of iron and ferritin most evident within the globus pallidus. Parkinsonism is a salient clinical feature of these three diseases of brain iron accumulation.

Increased levels of iron have been found within the substantia nigra at autopsy $y^{22-25}$ in IPD. MRI studies of IPD brains also demonstrate nigral iron deposition. ${ }^{26}$ While the deposition of iron in the brain is less dramatic in IPD than in the previously mentioned diseases, some evidence points towards iron toxicity as the primary insult to dopaminergic neurones. ${ }^{27} 28$ Three of the cases reported are still alive, and thus neuropathological evidence of brain iron overload is not available. As no autopsy was performed on the only mortality, case 2, there is no neuropathological confirmation of IPD in any of these cases.

The abnormal iron handling in $\mathrm{HH}$ results in excessive tissue iron deposition with resultant organ damage. The central nervous system is thought to be protected from excessive iron deposition because of "compartmentalisation" of systemic and brain iron stores. Despite this, there have been several case reports of neurological dysfunction in haemochromatosis patients, although whether these cases had genetically determined haemochromatosis is in doubt. Many early accounts were confounded by coexistent hepatic encephalopathy or alcoholism, which could, in part, explain some of the clinical features. ${ }^{5}{ }^{6}$ The first comprehensive catalogue of neurological problems directly attributable to the underlying haemochromatosis was a clinical review of 34 cases, ${ }^{7}$ which included changes in cognitive dysfunction, hearing loss, and peripheral neuropathy. Royden Jones described two patients with ataxia, rigidity, myoclonic jerks, and dementia, who were found to have haemochromatosis. There was no evidence of liver failure or other confounding factors. ${ }^{8}$ Other authors have reported small numbers of cases with parkinsonism and other neurological features including cerebellar, akinetic-rigid, dyskinetic and dementing syndromes. Nielsen ${ }^{3}$ described the development of a syndrome of dementia, dysarthria, progressive gait disturbance, ataxia, and parkinsonism in a young male with haemochromatosis. Brain MRI showed a pronounced signal decrease in the caudate nucleus, medial globus pallidus, and red nucleus. It was concluded that basal ganglionic iron deposition mediated the neurological decline. A second report also noted 
abnormal basal ganglia signal intensities on MRI. ${ }^{2}$ All these cases included neurological involvement outside the basal ganglia in contrast to ours, which are clinically typical IPD.

The function of the blood-brain barrier in $\mathrm{HH}$ is poorly studied, with little known of iron transport across it. In haemochromatosis, iron pigmentation of the pituitary gland, pineal body, leptomeninges, lentiform nucleus, dentate nucleus, choroid plexus, and paraventricular areas of the human brain is well documented. ${ }^{29}{ }^{30}$ A recent neuroradiological study revealed abnormal basal ganglia signal intensities on MRI in 6 of 14 neurologically normal individuals with $\mathrm{HH}$, suggesting excess iron deposition in the basal ganglia. ${ }^{1}$ There is increasing evidence for the traffic of iron across the blood-brain barrier by transferrin receptor mediated endocytosis of transferrin-iron complex, with subsequent release of iron, either free or bound to transferrin, into the brain interstitium. ${ }^{31} 32$ The blood-brain barrier is likely to regulate the ebb and flow of iron to and from the brain interstitial fluid and CSF in a dynamic fashion, probably by altering the number of transferrin receptors expressed on cerebral capillary endothelial cells. This challenges the view that the brain is impervious to iron from systemic tissues. Animal studies have not demonstrated excess iron deposition consequent to short term systemic iron overload. However, the chronic (over decades) systemic iron overload seen in human $\mathrm{HH}$ poses a major challenge for the blood-brain barrier in maintaining brain iron homeostasis.

We have described four cases of concurrent HH and IPD. The chronic systemic iron overload seen in $\mathrm{HH}$ may result in a spill over of iron through the blood-brain barrier into areas of the brain known to store iron, particularly the basal ganglia. We speculate that iron deposition might occur within susceptible brain areas in $\mathrm{HH}$, resulting in neurological illness. These cases of IPD occurring in individuals with $\mathrm{HH}$ add some weight to the possibility of iron toxicity as a significant factor in the aetiology of IPD. We acknowledge that both IPD and HH are common diseases and it is feasible that both conditions could affect any given individual by chance. We also acknowledge that parkinsonism per se rather than IPD would be more likely to occur if iron deposition were to occur within the basal ganglia in $\mathrm{HH}$.

Basal ganglia iron deposition is a feature of conditions simulating IPD such as multiple system atrophy and progressive supranuclear palsy but not IPD. We believe that the clinical cases described here represent probable IPD. Prospective studies examining the prevalence of IPD among genetically confirmed, iron overloaded HH individuals would further clarify any possible link between these two conditions. If a real association becomes apparent between $\mathrm{HH}$ and IPD, $\mathrm{HH}$ should be considered in the evaluation of IPD patients.

\section{Authors' affiliations \\ D J Costello, S L Walsh, H J Harrington, Department of Neurology and Medicine, Mercy Hospital, Grenville Place, Cork, Ireland \\ C H Walsh, Department of Endocrinology and Medicine, South Infirmary Hospital, Old Blackrock Road, Cork, Ireland \\ Competing interests: none declared}

Correspondence to: $\operatorname{Dr} \mathrm{C} \mathrm{H}$ Walsh, Department of Endocrinology and Medicine, South Infirmary Hospital, Old Blackrock Road, Cork, Ireland; hurley.phyl@sivh.ie

Received 3 September 2003

Accepted 3 September 2003

\section{REFERENCES}

1 Berg D, Hoggenmuller U, Hofmann E, et al. The basal ganglia in haemochromatosis. Neuroradiology 2000;42:9-13.

2 Schröder J, Haan J. Extrapyramidales Syndrom bei idiopathischer Hämochromatose (IHC). Nervenartz 1987;58:577-8.

3 Nielsen JE, Neerup Jensen L, Krabbe K. Hereditary haemochromatosis: a case of iron accumulation in the basal ganglia associated with a parkinsonian syndrome. J Neurol Neurosurg Psychiatry 1995;59:318-21.

4 Demarquay G, Setiey A, Morel Y, et al. Clinical report of three patients with hereditary haemochromatosis and movement disorders. Movement Dis 2000;15:1204-9.

5 Kuipers FC. Over Haemochromatosis met hepato-cerebral degeneratie. These de Univ U J Enschede \& Zonen Amsterdam Haarlem 1932.

6 Scully RE, Galdabini JJ, McNeely BU. Case records of the Massachusetts General Hospital: case 17-1979. N Engl J Med 1980;300:969-76.

7 Milder MS, Cook JD, Stray S, et al. Idiopathic haemochromatosis, an interim report. Medicine 1980;59:34-49.

8 Royden Jones Jr H, Hedley-Whyte ET. Idiopathic haemochromatosis (IHC): Dementia and ataxia as presenting signs. Neurology (Cleveland) 1983;33:1479-83.

9 Harvey RJ, Summerfield JA, Fox NC, et al. Dementia associated with haemochromatosis: a report of two cases. Eur J Neurol 1997:4:318-22.

10 Rajput AH, Offord KP, Beard CM, et al. Epidemiology of Parkinsonism: incidence, classification and mortality. Ann Neurol 1984; 16:278-82.

11 McDonnell SM, Preston BL, Jewell SA, et al. A survey of 2851 patients with haemochromatosis: symptoms and response to treatment. Am J Med 1999;106:619-24.

12 Riederer P, Dirr A, Goetz M, et al. Distribution of iron in different brain regions and subcellular compartments in Parkinson's disease. Ann Neurol 1992;32:S101-4.

13 Hallgren B, Sourander P. The effect of age on the nonhaemin iron in the human brain. J Neurochem 1958;3:41-51.

14 Octave JN, Schneider YJ, Trovet A, et al. Iron uptake and utilisation by mammalian cells. I: Cellular uptake of transferrin and iron. Trends Biochem Sci 1983;8:217-20.

15 Youdim MBH, Ben-Shachar D, Riederer P. Is Parkinson's disease a progressive siderosis of substantia nigra resulting in iron and melanin induced neurodegeneration? Acta Neurol Scand 1989;26:47-54.

16 Halliwell B. Reactive oxygen species and the central nervous system. J Neurochem 1992;59:1609-23.

17 Zhou B, Westaway SK, Levinson B, et al. A novel panthothenate kinase gene (PANK2) is defective in Hallervoden-Spatz syndrome. Nat Genet $2001 ; 28: 345-9$

18 Attieh ZH, Mukhopadhyay CK, Seshadri V, et al. Ceruloplasmin ferroxidase activity stimulates cellular iron uptake by a trivalent cation-specific transport mechanism. J Biol Chem 1999;274:1116-23.

19 Mukhopadhyay CK, Attiah ZK, Fox PL. Role of ceruloplasmin in cellular iron uptake. Science 1998;279:714-17.

20 Gitlin JD. Aceruloplasminemia. Pediatr Res 1998;44:271-6.

21 Curtis ARJ, Fey C, Morris CM, et al. Mutation in the gene encoding ferritin light polypeptide causes dominant adult-onset basal ganglia disease. Nat Genet $2001 ; 28: 350-4$.

22 Earle KM. Trace metals in Parkinson's disease. J Neuropathol Exp Neurol 1968;27:1-14.

23 Dexter DT, Wells FR, Lees AJ, et al. Increased nigral iron content in postmortem parkinsonian brain. Lancet 1987;2:1219-20.

24 Sofic $\mathbf{E}$, Riederer $\mathrm{P}$, Heinsen $\mathrm{H}$, et al. Increased iron (III) and total iron content in post-mortem substantia nigra of parkinsonian brain. J Neurol Transm 1988;74:199-205.

25 Riederer $\mathbf{P}$, Sofic E, Rausch W, et al. Transition metals, ferritin, glutathione, and ascorbic acid in parkinsonian brains. J Neurochem 1989;52:515-20.

26 Gorell JM, Ordidge RJ, Brown GG, et al. Increased iron-related MRI contrast in the substantia nigra in Parkinson's disease. Neurology 1995; $45: 1138-43$

27 Gerlach M, Ben-Shachar D, Riederer P, et al. Altered brain metabolism as a cause of neurodegenerative diseases? J Neurochem 1994;63: 793-807.

28 Ostrerova-Golts N, Petrucelli L, Hardy J, et al. The A53T $\alpha$-synuclein mutation increases iron-dependent aggregation and toxicity. J Neurosci 2000;20:6048-54

29 Cammermeyer J. Deposition of iron in paraventricular areas of the human brain in haemochromatosis. Neuropathol Exp Neurol 1947;2:111-27.

30 Sheldon JH. The iron content of the tissues in haemochromatosis, with special reference to the brain. QJ Med 1927;21:123-37.

31 Morgan EH. Iron and transition metal transport into the brain. Alfred Benzon symposium: brain barrier systems. 1999;45:357-70.

32 Moos T, Morgan EH. Brain iron homeostasis. Alfred Benzon symposium: brain barrier systems. 1999;45:371-86. 